\title{
Quantensprung oder Störenfried?
}

\section{Die Windkraftnutzung drängt aufs Meer. Doch die tatsächlichen Wirkungen von Windparks auf die Meeresumwelt sind noch weitgehend unbekannt. Ökologische Forschung und die Raumordnung werden damit zum Schlüssel für den naturverträglichen Ausbau der Offshore-Windkraftnutzung.}

$\mathrm{D}$ Von Bernd Neukirchen e Erfolgsgeschichte der Windkraftnutzung an Land wurde in Deutschland mit der gesetzlichen Abnahmeverpflichtung für Strom aus Erneuerbaren Energien und festgelegten Vergütungssätzen eingeleitet. Zum Zeitpunkt der Verabschiedung des Stromeinspeisungsgesetzes 1990 lag die installierte Gesamtleistung der Windenergie an Land noch bei 68 Megawatt, heute liegt sie bei über 15.300 .

Die Rahmenbedingungen für die Offshore-Windkraftnutzung wurden jedoch erst durch die immer knapper werdenden Landstandorte, die technischen Neuerungen der Anlagen und das Erneuerbare Energien Gesetz (EEG) entscheidend verbessert. Mit der am 1. August 2004 in Kraft getretenen Novelle des EEG werden weitere Anreize geschaffen, welche die wirtschaftliche Erschließung küstenferner Standorte auch nach 2010 sichern soll.

Trotz des mit diesen Regelungen ausgelösten Booms der Offshore-Windkraft sind in Nord- und Ostsee bislang weder im Zuständigkeitsbereich der Länder in der zwölf Seemeilen-Zone noch in der ausschließlichen Wirtschaftszone (AWZ; 12 bis 200 Seemeilen) Anlagen errichtet worden, einzelne Testanlagen in Küstennähe ausgenommen. Dennoch liegen allein für die AWZ, für die der Bund zuständig ist, derzeit 30 Anträge auf Genehmigung von Windkraftparks vor, 24 für die Nordsee, sechs für die 0stsee. Sechs der Vorhaben in der Nordsee wurden bereits genehmigt. In den Küstenländern befinden sich derzeit vier Pilotvorhaben in Planung.

Die Bundesregierung misst der seewärtigen Windenergienutzung ein enormes Entwicklungspotential bei. In einer ersten Bauphase bis 2006 sollen insgesamt mindestens 500 MW und bis 2010 zwei bis drei Gigawatt Leistung auf See installiert werden. Langfristig, das heißt bis 2025 beziehungsweise 2030, werden bei Erreichen der Wirtschaftlichkeit etwa 20.000 bis 30.000 Megawatt installierter Leistung für möglich gehalten (1).

\section{Offshore-Windkraft und Naturschutz}

Zusätzlich zum Klimaschutz soll die Windkraft auch einen wichtigen Beitrag zur Bewahrung der Natur und der biologischen Vielfalt leisten. Das gelingt jedoch nur, wenn die räumliche Planung der Windkraftnutzung sorgfältig mit Naturschutzbelangen abgestimmt wird. Der Konflikt zwischen Klimaschützern auf der einen und Naturschützern auf der anderen Seite muss deshalb zugunsten einer naturverträglichen Standortplanung und einer erhöhten Akzeptanz der Windenergie vermieden werden.

Grundsätzlich sind die von den Anlagen ausgehenden Wirkungen an Land und auf See die gleichen: direkte Flächenbeanspruchung, Vertreibungs- und Scheucheffekte beim Bau und Betrieb der Anlagen, Vogelschlagrisiko, Schallemissionen, Beeinträchtigung des Landschaftsbildes. Im Vergleich zu terrestrischen Lebensräumen sind die Kenntnisse über die marine Ökologie und die Ansprüche einzelner Arten jedoch eher gering. Die tatsächlichen Auswirkungen sind deshalb nicht nur schwerer zu bewerten, sondern auch die notwendigen Untersuchungen im Rahmen der Umweltverträglichkeitsplanung (UVP) sind methodisch und zeitlich aufwändiger.

Sandbänke und Riffe - nach Anhang I der FloraFauna-Habitat-Richtlinie (FFH-RL) besonders geschützte Lebensraumtypen - können durch Überbauung verloren gehen. Sedimentumlagerungen, künstliche Riffeffekte oder Erwärmung des Meeresbodens durch Energieverluste der Stromkabel können Benthosgemeinschaften beeinträchtigen. Schallimmissionen, insbesondere während der Rammarbeiten zur Errichtung der Anlagen, können Flucht- und Meidereaktionen von Meeressäugetieren auslösen und zu dauerhaften Hörschäden und Habitatverlusten führen. Die Wirkungen auf Schweinswale, die ebenfalls unter dem strengen Schutz der FFH-RL (Anhang II) stehen, sind dabei besonders sorgfältig zu be- trachten. Zug- und Rastvögel können durch Vogelschlag oder Barrierewirkungen gefährdet werden; zudem können die Flächen der Windparks aufgrund von Meidereaktionen als Lebensraum teilweise oder ganz verloren gehen (2).

Erste Erkenntnisse über die tatsächlichen Auswirkungen größerer Offshore-Windenergieparks liegen bisher allerdings nur aus Dänemark vor. Die 80 beziehungsweise 72 Einzelanlagen der Windparks „Horns Rev“ in der Nordsee und „Nysted" in der Ostsee liegen maximal bis zu 20 Kilometern von der Küste entfernt in Wassertiefen zwischen 5 und 15 Meter.

Die bisherigen ökologischen Begleituntersuchungen aus der Bau- und Betriebsphase am Windpark Horns Rev zeigen aber bereits, dass an den festen Gründungsstrukturen wie erwartet eine Sukzession der Lebensgemeinschaften einsetzt und Fische die Strukturen als Rückzugs- und Nahrungsgebiet nutzen. Zu den Auswirkungen auf Schweinswale gibt es aus der schallintensiven Bauphase deutliche Hinweise auf Fluchtreaktionen, aber noch keine eindeutig interpretierbaren Ergebnisse für die Betriebsphase. Bei Seetauchern scheint sich die Vermutung, dass sie das Gebiet vollständig meiden, zu bestätigen. Ergebnisse zum Vogelschlagrisiko liegen noch nicht vor. Eine ausführliche Darstellung und Diskussion der Ergebnisse wird auf einer wissenschaftlichen Tagung im September 2004 in Dänemark erwartet.

\section{Räumliche Steuerung zur Konfliktlösung}

Zahlreichen Forschungsvorhaben des Bundes werden die Grundlagen für die räumliche Planung und die Bewertung der tatsächlichen Auswirkungen von Offshore-Windkraft verbessern. Zudem werden Datenlücken durch die Umweltverträglichkeitsuntersuchungen im Rahmen der Genehmigungsverfahren und später durch die obligatorischen Begleituntersuchungen beim Bauund Betrieb der Anlagen geschlossen. Insgesamt wird damit die Basis für langfristig sinnvolle Szenarien und Strategien zur räumlichen Steuerung und zur Ableitung von Vermeidungs- und Verminderungsmaßnahmen geschaffen.

Erste wichtige Schritte zur räumlichen Steuerung wurden im Mai 2004 mit der Meldung von Natura 2000-Gebieten in der AWZ an die EU-Kommission und mit den vom Bundesamt für Seeschifffahrt und Hydrographie (BSH) eingeleiteten Verfahren zur Ausweisung von , besonderen Eignungsgebieten für Windkraftanlagen“ nach $\S 3 a$ SeeAnlV getan (3). Trotzdem kommt beiden Gebietskate- 


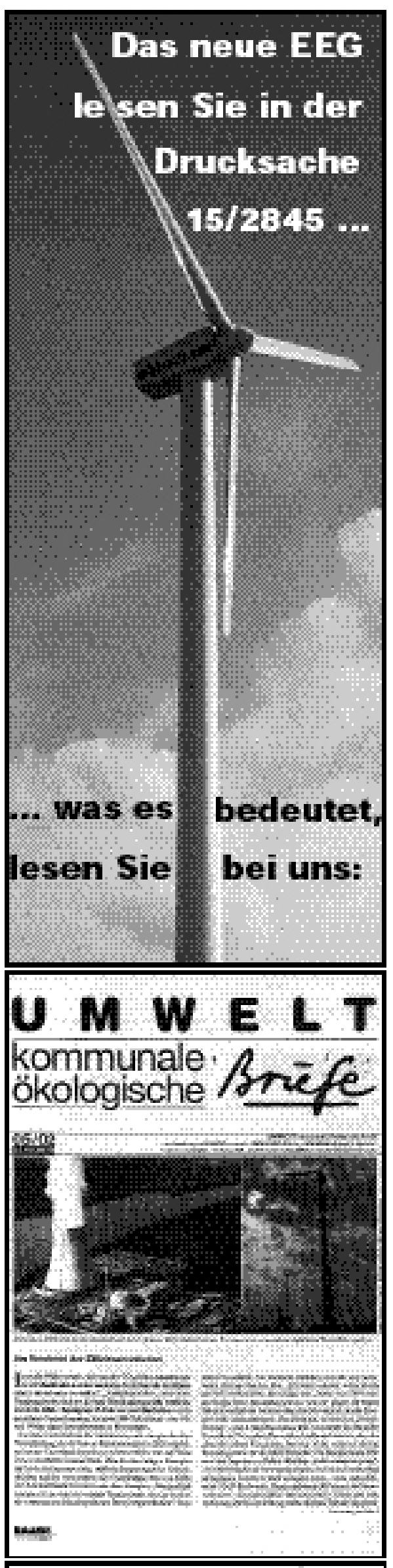

www.umweltbriefe.de gorien bisher nur eine verhältnismäßig schwache Steuerungswirkung zu. Natura-2000-Gebiete können aufgrund der erhöhten Anforderungen an die Anlagenzulassung zwar dazu führen, dass erst gar keine Anträge in diesen Gebieten gestellt werden, eine Verpflichtung dazu besteht allerdings nicht. Eine steuernde Wirkung können sie nur dann entfalten, wenn die Prüfung der FFH-Verträglichkeit im konkreten Genehmigungsverfahren negativ ausfällt und das Vorhaben damit unzulässig ist. Wenn zwingende Gründe des überwiegenden öffentlichen Interesses vorliegen und keine zumutbaren Alternativen gegeben sind, kann dennoch eine Genehmigung erteilt werden.

Eignungsgebiete nach $\S 3 a$ SeeAnlV entfalten im Gegensatz zu Eignungsgebieten im Rahmen der Raumordnung ( $\$ 7$ Abs. 4 Nr. 3 ROG), die mit einer Ausschlusswirkung an anderer Stelle verbunden sind, ebenfalls keine bindende Wirkung. Diese unter anderem vom Rat von Sachverständigen für Umweltfragen (SRU) geäußerte Kritik wird mit der Ausdehnung der Raumordnung auf die AWZ im Rahmen des Europarechtsanpassungsgesetzes Bau beseitigt (4). Dann können nicht nur die Windparkplanungen jenseits der Zwölf-Seemeilen-Zone räumlich gesteuert werden, sondern auch Kabeltrassen für die Anbindung der Offshore-Windparks an das Stromnetz festgelegt werden.

Ein wirksames Steuerungselement wurde allerdings bereits mit der Novelle des EEG eingeführt. In $\S 10$ (7) EEG wird die Förderung für OffshoreWindkraftanlagen in Schutzgebieten innerhalb der AWZ und in der Zwölf-Seemeilen-Zone beschränkt. Strom aus Anlagen, die innerhalb von Schutzgebieten liegen, wird nur noch dann gesetzlich vergütet, wenn sie vor dem 1.1.2005 genehmigt werden/ worden sind. Danach genehmigte Anlagen wären wahrscheinlich unwirtschaftlich. Die Regelung führt damit voraussichtlich zum Verzicht auf weitere Planungen innerhalb der besonders sensiblen Naturräume.

\section{Perspektiven}

Sechs Vorhaben in der AWZ der Nordsee mit insgesamt über 400 Einzelanlagen und einer installierbaren Gesamtleistung von mehr als 1500 MW sind bereits genehmigt. Die in Anspruch genommene Fläche würde sich auf über 170 Quadratkilometer belaufen. Weitere 13 Vorhaben sind im Verfahren bereits soweit vorangeschritten, dass noch in diesem Jahr mit weiteren Entscheidungen des Bundesamtes für Seeschifffahrt und Hydrographie (BSH) gerechnet werden kann. Wind- parkgrößen mit über 80 Einzelanlagen werden in der ersten Baustufe vom BSH derzeit nicht als genehmigungsfähig angesehen. Weitere Beschränkungen gibt es wegen des Anspruches auf Erteilung der Genehmigung - wenn die Sicherheit und Leichtigkeit des Verkehrs nicht beeinträchtigt und die Meeresumwelt nicht gefährdet wird - nicht. Ein planerischer Abwägungsspielraum besteht ebenfalls nicht. Gerade deshalb ist die Regelung über den Ausschluss der Einspeisevergütung in Schutzgebieten im Vorgriff auf die räumliche Planung sehr zu begrüßen.

Wird dieser eingeschlagene Weg der räumlichen Steuerung konsequent fortgeführt, sind die aufgezeigten Konflikte hinsichtlich der Offshore-Windkraft lösbar. Ob sich die zum Teil erheblichen Auseinandersetzungen mit anderen Nutzungen im Zuge der Raumordnung ebenfalls bewältigen lassen, sei dahin gestellt. Die Offshore-Windkraft vor diesem Hintergrund als Störenfried zu bezeichnen, ist nicht gerechtfertigt. Aber auch von einem Quantensprung kann - trotz der rasanten Entwicklung - erst dann gesprochen werden, wenn die zahlreichen technischen Herausforderungen neuer Anlagentypen, neuer Gründungstechniken, der Kabelanbindung und der Netzeinspeisung bewältigt werden, die notwendige Wirtschaftlichkeit erreicht wird und die naturverträgliche Erschließung möglicher Offshore-Standorte langfristig sichergestellt ist.

\section{Anmerkungen}

(1) Bundesministerium für Umwelt, Naturschutz und Reaktorsicherheit ( $\mathrm{Hg}$.): Strategie der Bundesregierung zur Windenergienutzung auf See. Berlin 2002.

(2) UBA (Hg.): Offshore-WEA, Abschlussbericht zum F\&E Vorhaben 20097106 „Untersuchungen zur Vermeidung und Verminderung von Belastungen der Meeresumwelt durch Offshore-Windenergieanlagen im küstenfernen Bereich der Nord- und Ostsee", 30. Juni 2003, Berlin 2003.

(3) www.habitatmarenatura2000.de

(4) Rat von Sachverständigen für Umwelffragen ( $\mathrm{Hg}$. ): Windenergienutzung auf See. Stellungnahme, Berlin 2003.

Der Autor
Bernd Neukirchen ist Leiter des Leistungs-
schwerpunkts Offshore-Windkraftnutzung beim
Bundesamt für Naturschutz (BfN).
Kontakt: BfN, Konstantinstrasse 110,
3179 Bonn. Tel. 0228-8491236,
E-Mail: Bernd.Neukirchen@bfn.de


(c) 20I0 Authors; licensee IÖW and oekom verlag. This is an article distributed under the terms of the Creative Commons Attribution Non-Commercial No Derivates License (http://creativecommons.org/licenses/by-nc-nd/3.o/), which permits unrestricted use, distribution, and reproduction in any medium, provided the original work is properly cited. 